\title{
Wavelength Tunable 10-GHz 3-ps Pulse Source Using a Dispersion Decreasing Fiber-Based Nonlinear Optical Loop Mirror
}

\author{
Ju Han Lee, Student Member, IEEE, Taichi Kogure, Member, IEEE, and David J. Richardson
}

\begin{abstract}
We experimentally demonstrate the use of a dispersion decreasing fiber (DDF)-based nonlinear optical loop mirror (NOLM) for the generation of wavelength tunable soliton-like pulses at a repetition rate of $10 \mathrm{GHz}$. We compress $\sim 12-\mathrm{ps}$ Gaussian pulses from an electro-absorption modulator (EAM) (followed by $125 \mathrm{~m}$ of DCF for preliminary linear dispersion compensation) into 3-ps pedestal-free pulses using both high-order soliton compression and nonlinear switching effects within an 8.5-km DDF-based loop mirror. The output pulses from the DDF-based NOLM show considerable pedestal reduction compared to those obtained by directly compressing the EAM seed pulses via a single passage through the DDF. Wavelength tuning of the compressed pulses over a 15-nm bandwidth (from 1541 to $1556 \mathrm{~nm}$ ) is demonstrated without a significant increase in pulse duration or degradation in pulse quality.
\end{abstract}

Index Terms-Nonlinear optical loop mirror, nonlinear optics, optical pulse compression, optical solitons, optical switching.

\section{INTRODUCTION}

$\mathbf{T}$ HE drive to develop all-optical time division multiplexing (OTDM) systems capable of operating at data rates in excess of $40 \mathrm{~Gb} / \mathrm{s}$ has resulted in great interest in the development of stable sources of high-repetition rate ultrashort optical pulses operating in the 1550-nm telecommunications band. Indeed, single-channel data rates in excess of $1 \mathrm{~Tb} / \mathrm{s}$ have now been demonstrated requiring the generation of pulses of subpicosecond duration [1]. The direct generation of high-quality short pulses of a few picosecond duration at a repetition rate of $\sim 10 \mathrm{GHz}$ (the base repetition rate typically used to date for OTDM applications) is difficult to achieve in practice and the conventional approach has been to use an active harmonically mode-locked fiber-ring laser [2]. This approach allows for the generation of high-quality transform limited pulses and subpicosecond pulse generation has been achieved in this way. However, such lasers are complex and tend to suffer from environmental instability problems unless constructed from polarization maintaining components. Moreover, active control of the cavity length is required in order to accurately maintain the

Manuscript received June 20, 1993; revised September 22, 2003.

J. H. Lee and D. J. Richardson are with the Optoelectronics Research Centre, University of Southampton, Southampton SO17 1BJ, U.K. (e-mail j.h.lee@ieee.org).

T. Kogure is on leave from the Information Technology R\&D Center, Mitsubishi Electric Corporation, Kamakura 247-8501, Japan.

Digital Object Identifier 10.1109/JSTQE.2003.822911 pulse repetition rate at a specific well defined frequency. An alternative approach to high-repetition rate pulse generation is to use a fast optical modulator such as an electro-absorption modulator (EAM) to create a train of somewhat longer pulses, typically $10-15$ ps and limited by the modulation bandwidth, and to then use some form of pulse compression technique to obtain the shorter pulse durations required [3]. This approach provides for very robust and practical optical sources; however, the quality of the final pulses is often compromised in the compression process and this can lead to severe issues when used for demanding applications such as OTDM. The development of effective pulse compression techniques capable of providing high-quality pulse output (often from a chirped pulse input) is thus an important issue and has received much research interest over recent years.

Adiabatic soliton pulse compression has long been considered a promising and simple technology for the generation of high-quality short-duration pulse trains [4]. A range of adiabatic pulse compression techniques have been experimentally demonstrated and these techniques were based on either dispersion profiled fibers (dispersion decreasing fiber, comb-like dispersion profiled fiber, and step-like dispersion profiled fiber) [5]-[7] or uniform fibers with distributed Raman amplification [4], [8]. Although this technology provides a powerful approach to generate soliton-like short duration pulses, small departures from the required adiabatic condition along the fiber length due to either nonoptimized dispersion variations along dispersion profiled fiber [9], or excessive Raman gain variations along each soliton period of distributed Raman amplifier compressors [10], lead to the growth of broad low-level pulse pedestals, which are detrimental for ultrahigh-speed OTDM systems application. In order to obtain high-quality short duration pulses with a suppressed pulse pedestal, the use of a nonlinear optical switch after the adiabatic pulse compression process was suggested and successfully demonstrated experimentally, e.g., using a dispersion shifted fiber (DSF)-based nonlinear optical loop mirror (NOLM) [11] or dispersion flattened fiber (DFF)-based dispersion imbalanced nonlinear optical loop mirror (DI-NOLM) [12]. Several research groups have proposed pulse compression schemes involving the use of dispersion profiled fibers within DI-NOLM schemes to obtain simultaneous soliton pulse compression and pedestal removal [13]-[15]. For example, Tadakuma et al. [13] experimentally demonstrated short-pulse generation using a comb-like dispersion profiled fiber-based DI-NOLM. In 1996, Evans [14] and 


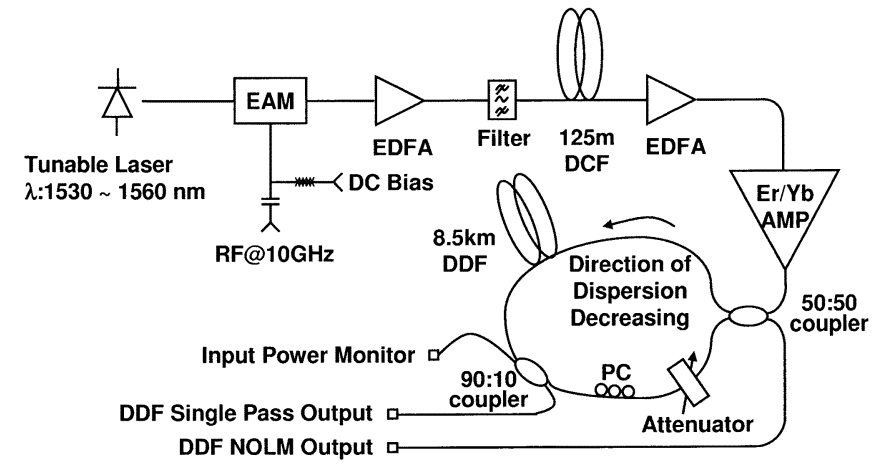

Fig. 1. Experimental setup for the tunable pulse source using a DDF-based NOLM.

more recently Cao et al. [15] suggested the use of a DDF-based DI-NOLM to obtain both soliton pulse compression and nonlinear switching, and theoretically demonstrated the possibility of generating pedestal-free soliton-like pulses.

In this paper, we experimentally demonstrate a DDF-based NOLM for simultaneous soliton pulse compression and pedestal removal and show results concerning the successful generation of 10-GHz wavelength tunable pedestal-suppressed short-duration pulses. Our scheme differs in operation from those previously proposed in [14] and [15] and incorporates a strategically positioned attenuator within the NOLM to ensure high-quality pulse compression. We compress 12-ps Gaussian pulses from an EAM (followed by an 125-m DCF) into 3-ps pedestal-free soliton-like pulses using an $8.5-\mathrm{km}$ DDF-based loop mirror. The output pulses are then compared to those directly generated from the DDF without a loop mirror configuration in terms of pulse pedestal level. Wavelength tunability over $\sim 15 \mathrm{~nm}$ is readily achieved without appreciable degradation in pulse quality.

\section{EXPERIMENTAL SETUP AND THEORETICAL MOdELING FOR THE DISPERSION DECREASING FIBER NOLM-BASED PULSE SOURCE}

Our experimental setup for the DDF NOLM-based tunable pulse source is shown in Fig. 1. A continuous wave (CW) external cavity laser tunable in the range of $1530-1560 \mathrm{~nm}$ was used as the input seed source. The CW light was launched into the input port of a commercially available EAM to generate Gaussian-shaped pulses. The EAM was driven electrically with a dc bias of up to $6 \mathrm{~V}$ and a 10-GHz sinusoidal radio-frequency (RF) modulation [3]. Fig. 2(a) shows the extinction ratio of the EAM as a function of reverse bias voltage at various operating wavelengths. From Fig. 2(a), it is clear that by properly setting the bias voltage between 3 and $5 \mathrm{~V}$ we can generate Gaussian shaped pulses with good interpulse extinction ratio $(>20 \mathrm{~dB})$ at a wavelength in the range $1530-1560 \mathrm{~nm}$. The pulses emerging from the EAM were first amplified in an erbium-doped fiber amplifier (EDFA) and passed though a 1-nm band pass filter before being passed through a DCF to compensate residual linear chirp generated in the EAM. The duration of the pulses after the EAM was $\sim 15 \mathrm{ps}$ and the pulses were compressed to $\sim 12$-ps duration at the DCF output. The time-bandwidth product of the pulses at the EAM output was

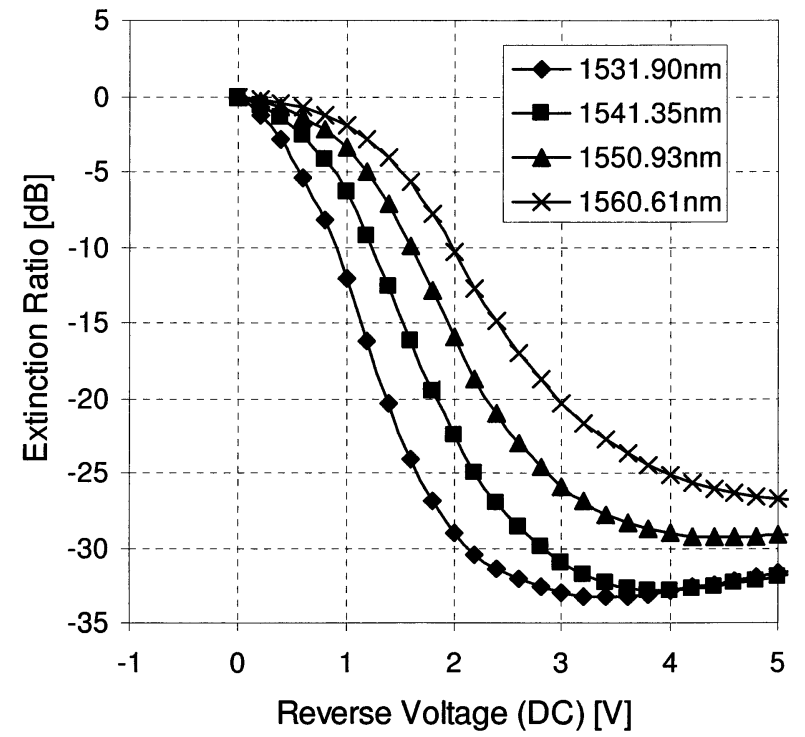

(a)

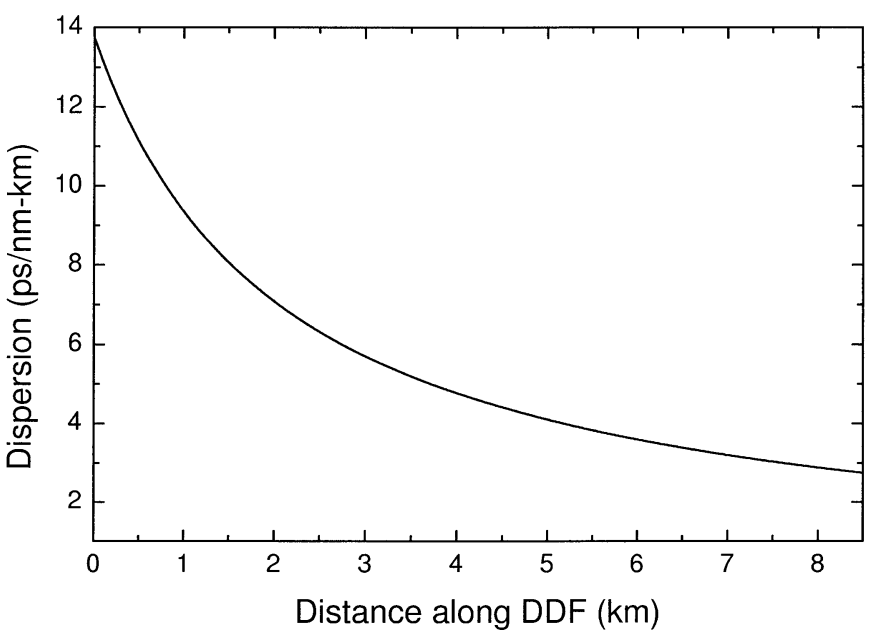

(b)

Fig. 2. (a) Measured extinction ratio of the EAM used in this experiment as a function of reverse bias voltage at various operating wavelengths. (b) Dispersion profile of the DDF used in this experiment.

$\sim 0.41$ meaning that the pulses were close to transform limited at this point in the system. These pulses were amplified up to a $23-\mathrm{dBm}$ average power with a high-power $\mathrm{Er} / \mathrm{Yb}$ amplifier and were then passed through the DDF-based NOLM in order to compress the pulsewidth and filter out the low-level pedestal associated with high-order soliton compression process in the DDF. The NOLM was constructed using a 50:50 coupler and an 8.5-km DDF. A 90:10 coupler was also inserted within the loop to allow us to directly monitor pulses after a single pass through the DDF (in the dispersion decreasing direction). The DDF dispersion profile (D) followed a hyperbolic profile at $1550-\mathrm{nm}$ tapering along the $8.5-\mathrm{km}$ length from 13.75 to $2.75 \mathrm{ps} / \mathrm{nm}-\mathrm{km}$ as shown in Fig. 2(b). This DDF was, in fact, originally designed and fabricated to adiabatically compress 40-GHz sinusoidal optical signals down to transform-limited $\sim 5$-ps fundamental soliton pulses. Further details concerning the characterization and fabrication of this DDF are provided in [16]. The dispersion profile of this fiber means that it is suitable for the "adiabatic" compression of $\sim 15$-ps Gaussian 
pulses down to $\sim 5$-ps solitons. However, using higher order soliton compression effects we were able to achieve further pulse compression down to our target pulsewidth of $\sim 3$ ps in a single pass through the fiber [17]. These pulses exhibit a small pedestal which needs to be suppressed for $80-\mathrm{Gb} / \mathrm{s}$ OTDM applications. The NOLM also incorporates a variable optical attenuator (VOA) positioned and adjusted to prevent pulses propagating in the dispersion increasing direction from undergoing significant soliton evolution as they pass through the loop, which we found otherwise compromised the quality of the compressed pulses. Note that pulses propagating in the dispersion increasing direction needed to be attenuated relatively strongly $(\sim 8 \mathrm{~dB})$ to avoid soliton effects due to the relatively small dispersion value and dispersion variation (2.75-5 ps/nm-km over 4-km fiber length). As a matter of fact, the dispersion decreasing fiber-based NOLM demonstrated in this paper is closer to a NOLM than a DI-NOLM and makes our device operation quite different from that theoretically proposed in [14] and [15].

In order to understand pulse evolution in the DDF and validate this system concept, the above system was modeled as a function of key parameters of the NOLM, for example coupling ratio, dispersion, length, attenuation, and the peak power of the input pulses which we assumed to be transform limited Gaussian pulses with 12-ps temporal width. Pulse propagation within the NOLM was modeled using the nonlinear Schrödinger equation incorporating terms to describe the effects of group velocity dispersion and self-phase modulation as described by

$$
\frac{\partial A}{\partial z}+\beta_{1} \frac{\partial A}{\partial t}+\frac{i}{2} \beta_{2} \frac{\partial^{2} A}{\partial t^{2}}-\frac{1}{6} \beta_{3} \frac{\partial^{3} A}{\partial t^{3}}+\frac{\alpha}{2} A=i \gamma|A|^{2} A
$$

where the pulse amplitude $A$ is normalized such that $|A|^{2}$ represents the optical field strength within the fiber. $\beta_{1}$ is the group delay, $\beta_{2}$ is the first-order group velocity dispersion (GVD), which is a function of the fiber distance along the DDF, and $\beta_{3}$ is the second-order GVD, which is assumed to be constant along the DDF length. $\alpha$ represents the absorption coefficient of optical power in the fiber, and $\gamma=n_{2} \omega_{o} / c A_{\text {eff }}$ is the nonlinearity coefficient where $n_{2}=2.6 \times 10^{-20} \mathrm{~m}^{2} / \mathrm{W}, \omega_{o}$ is the signal frequency, and $A_{\text {eff }}$ is the effective area of the fiber which varies along the fiber length in the range of $61 \sim 74 \mu \mathrm{m}^{2}$. We solved the above equation using the symmetrized split step Fourier method [18]. The value of dispersion slope $(d D / d \lambda)$, was $0.07 \mathrm{ps} / \mathrm{nm}^{2} / \mathrm{km}$ at a wavelength of $1550 \mathrm{~nm}$. The fiber loss was measured to be $0.3 \mathrm{~dB} / \mathrm{km}$.

\section{NumERICAL SimUlation AND EXPERIMENTAL RESUlTS}

In Fig. 3, the numerically predicted and experimentally measured power transmission characteristic of the DDF-based NOLM is plotted as a function of input average power at a wavelength of $1550 \mathrm{~nm}$. A good nonlinear switching characteristic for the NOLM, together with excellent agreement between theory and experiment, is clearly shown in the graph. From the nonlinear transmission curve in Fig. 3, the optimum system operating point from a nonlinear switching perspective would appear to be the region of $60-\mathrm{mW}$ input average power, but this operating point was found to provide $\sim 5$-ps duration

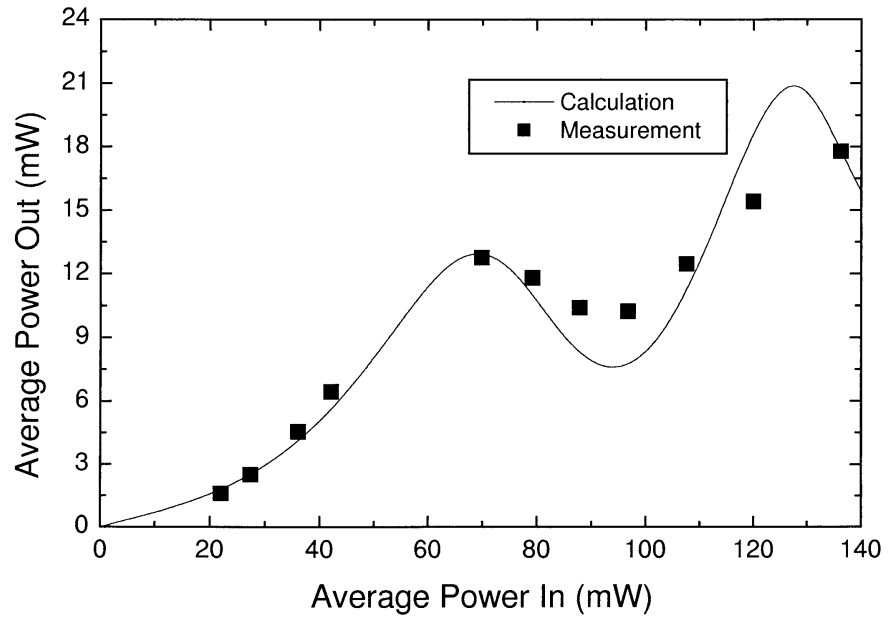

Fig. 3. Numerically predicted and experimentally measured power transmission characteristic of the DDF-based NOLM as a function of input pulse average power at a wavelength of $1550 \mathrm{~nm}$.

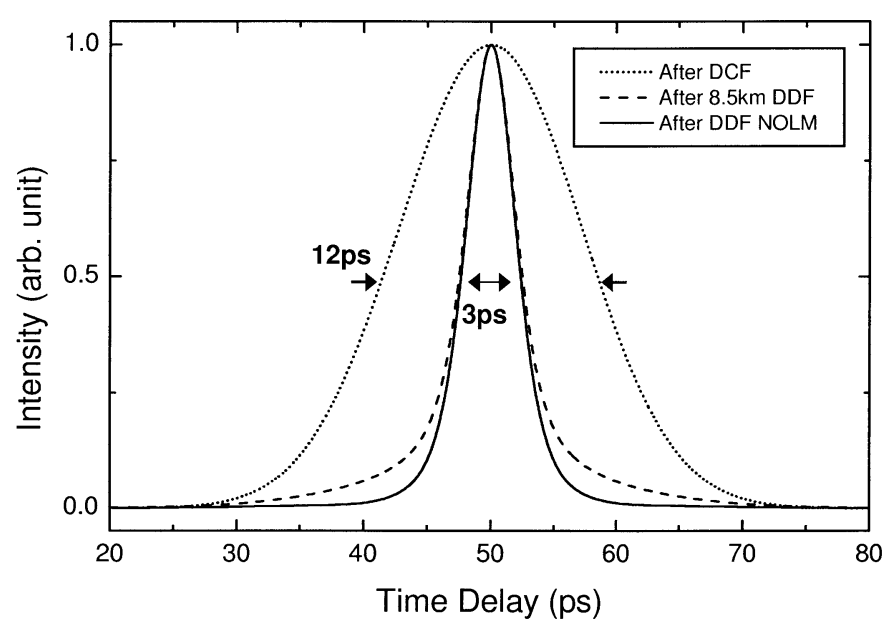

(a)

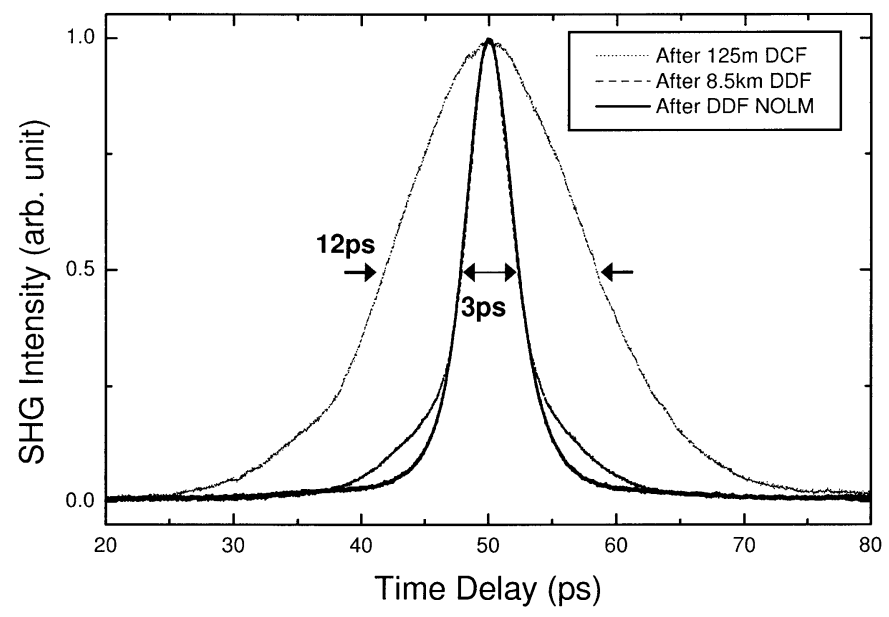

(b)

Fig. 4. (a) Theoretically predicted and (b) experimentally measured SHG autocorrelation functions of the compressed pulses both after the 8.5-km DDF single pass only and after the DDF-based NOLM at an operating wavelength of $1550 \mathrm{~nm}$ under the optimum operating condition of $\sim 120-\mathrm{mW}$ input pulse power, together with those of 12-ps Gaussian pulses generated with the EAM followed by the 125 -m-long DCF. 


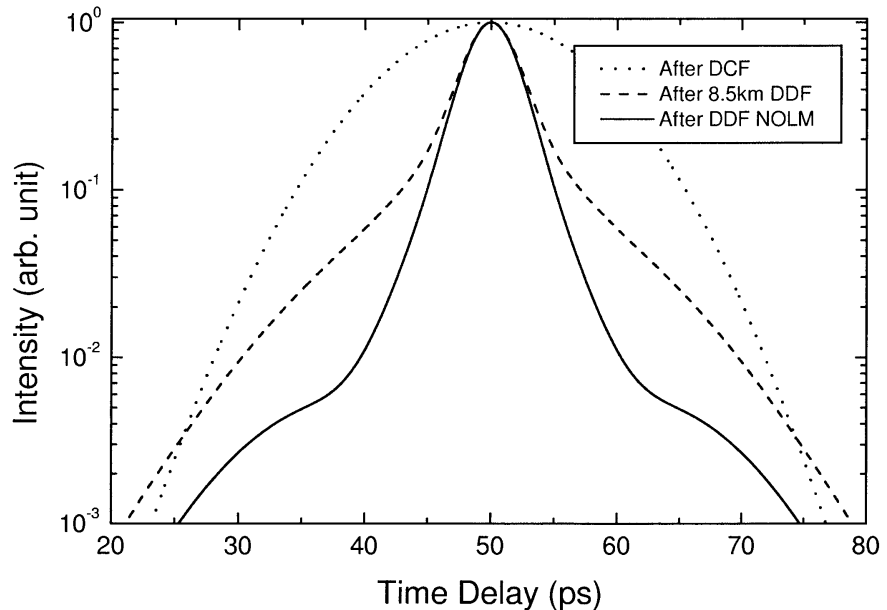

(a)

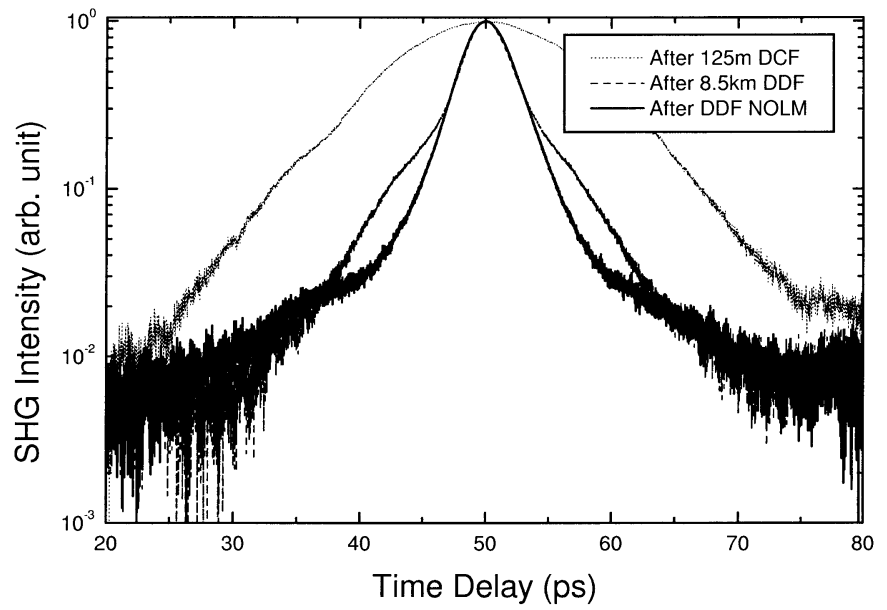

(b)

Fig. 5. Same data as Fig. 4 plotted in a log scale. (a) Theoretically predicted and (b) experimentally measured SHG autocorrelation traces.

pulses, which is twice broader than our target pulse duration of 3 ps. Both numerically and experimentally, we found the optimum system operating point for 3-ps pulse generation with maximum pedestal suppression to be in the region of $\sim 120 \mathrm{nW}$ of input power. Note that the agreement between theory and experiment is worse at higher powers, which we attribute to a slight degradation in our high-power (up to $28 \mathrm{dBm}$ ) amplifier performance due to buildup of out-of-signal-band ASE at the these higher gain levels.

Fig. 4 shows the theoretical and experimental SHG autocorrelation functions of the compressed pulses both after a single pass at the $8.5-\mathrm{km}$ DDF and after the DDF-based NOLM at an operating wavelength of $1550 \mathrm{~nm}$ under the optimum operating condition. Most of the 12-ps Gaussian pulses generated with the EAM followed by the DCF are also shown. Fig. 5 is a $\log$ scale plot of the same data which emphasizes the pedestal suppression provided by the approach. Although the final compressed pulsewidths were 3 ps after a single pass through the 8.5-km DDF and after the DDF-based NOLM, significant pulse pedestal was observed both theoretically and experimentally without the loop mirror structure, which can be attributed to the higher order soliton compression effect [17] needed to get this large a compression factor from this dispersion profile. By

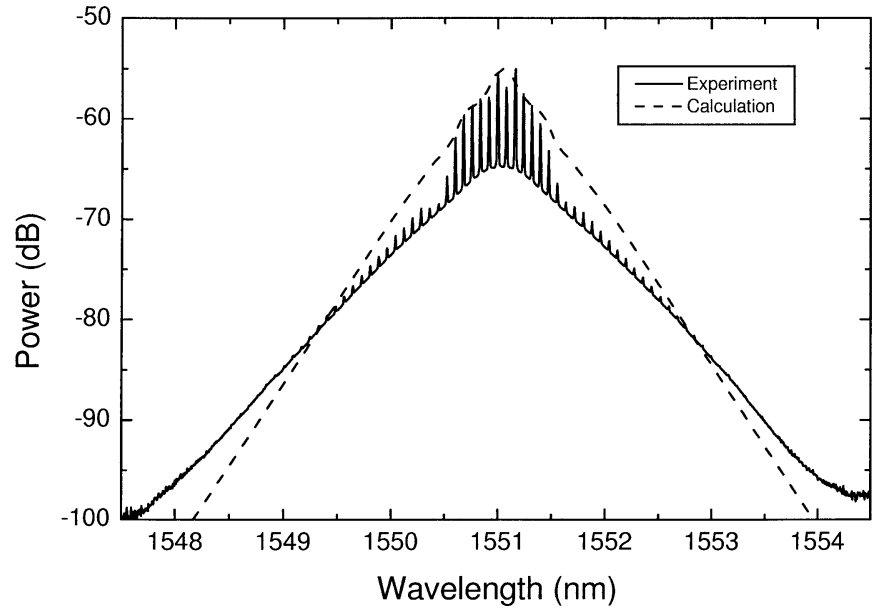

Fig. 6. Calculated and experimentally measured optical spectrum of the pulses from the DDF-based NOLM at an operating wavelength of $1550 \mathrm{~nm}$ under the optimum operating condition of $\sim 120$-mW input pulse power.

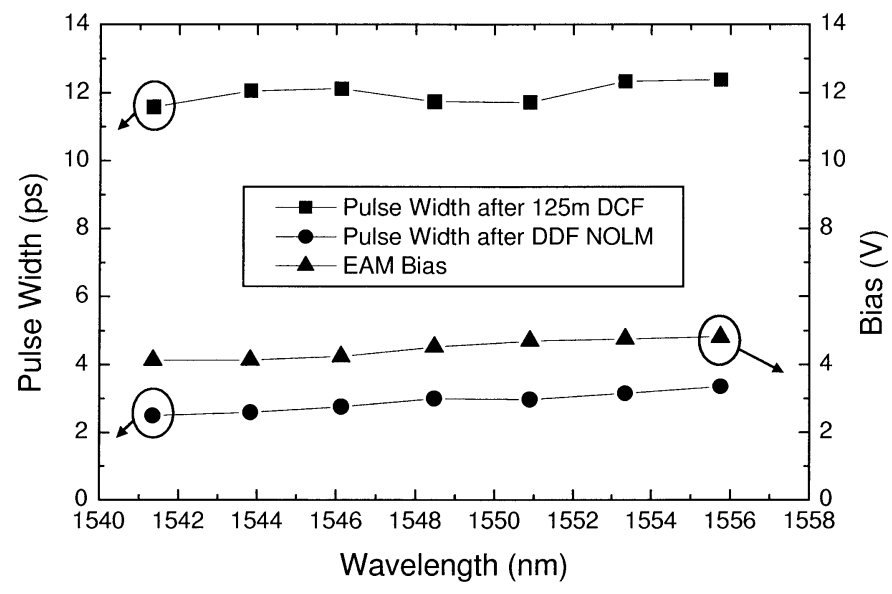

Fig. 7. Measured output pulsewidth from the DDF-based NOLM, together with the bias voltage versus wavelength at a fixed input Gaussian pulse average power of $\sim 120 \mathrm{~mW}$.

contrast, the DDF-based NOLM generated high-quality clean soliton-like pulses as shown in Figs. 4 and 5. It is clear from these results that the DDF-based NOLM function provides both high order soliton compression and nonlinear switching as predicted. The corresponding optical spectrum of the pulses from the DDF-based NOLM are shown in Fig. 6. The time-bandwidth product of the pulses was found to be less than $\sim 0.4$ (assuming a hyperbolic pulse shape for the pulsewidth estimation) and confirming that the pulses are of a good quality.

In order to characterize the wavelength tuning range of our DDF NOLM-based pulse source, we performed SHG autocorrelation measurements of the output pulses by changing the wavelength of the $\mathrm{CW}$ seed source. The results are summarized in Fig. 7. High-quality $\sim 3$-ps duration soliton-like pulses were obtained over a bandwidth of $\sim 15 \mathrm{~nm}$. Note that the temporal pulsewidth was found to increase very slightly toward longer wavelengths and that we had to increase the EAM bias voltage at these longer wavelengths to find the shortest pulsewidth and optimal pedestal suppression. This change of bias voltage can be attributed to the wavelength dependence of the residual chirp of the pulses generated from the EAM used in this experiment. 


\section{CONCLUSION}

We have experimentally demonstrated that the DDF-based nonlinear optical loop mirror can be used for the successful generation of $10-\mathrm{GHz}$ wavelength tunable soliton-like pulses. We generated high-quality 3-ps pedestal-free pulses from chirp compensated 12-ps Gaussian input seed pulses using the combined effects of higher order soliton compression and nonlinear switching in an 8.5-km DDF-based loop mirror. Tuning of the compressed pulses over a $\sim 15$-nm wavelength range was also readily achieved. A well-optimized DDF-based NOLM could be a powerful way to obtain high-quality ultrashort soliton-like pulses from robust pulse carving seed sources capable of generating only relatively broad Gaussian pulses.

\section{REFERENCES}

[1] M. Nakazawa, "Tb/s OTDM technology," in Proc. 27th Eur. Conf. Optical Communication (ECOC'2001), 2001, Paper Tu.L.2.3, pp. 184-187.

[2] K. K. Gupta, D. Novak, and H. Liu, "Noise characterization of a regeneratively mode-locked fiber ring laser," IEEE J. Quantum Electron., vol. 36, pp. 70-78, Jan. 2000.

[3] S. V. Chernikov, M. J. Guy, J. R. Taylor, D. G. Moodie, and R. Kashyap, "Duration-tunable 0.2-20 ps $10 \mathrm{GHz}$ source of transform-limited optical pulses based on an electroabsorption modulator," Opt. Lett., vol. 20, pp. 2399-2401, 1995.

[4] K. Igarashi, H. Tobioka, S. Takasaka, S. Matsushita, and S. Namiki, "Duration-tunable $100 \mathrm{GHz}$ sub-picosecond soliton train generation through adiabatic Raman amplification in conjunction with soliton reshaping," in Proc. Optical Fiber Communication Conf. (OFC'2003), 2003, Paper TuB6.

[5] S. V. Chernikov, D. J. Richardson, E. M. Dianov, and D. N. Payne, "Picosecond soliton pulse compression based on dispersion decreasing fiber," Electron. Lett., vol. 28, pp. 1842-1844, 1992.

[6] E. A. Swanson and S. R. Chinn, "40-GHz pulse train generation using soliton compression of a Mach-Zehnder modulator output," IEEE Photon. Technol. Lett., vol. 7, pp. 114-116, Jan. 1995.

[7] S. V. Chernikov, J. R. Taylor, and R. Kashyap, "Experimental demonstration of step-like dispersion profiling in optical fiber for soliton pulse generation and compression," Electron. Lett., vol. 30, pp. 433-434, 1994.

[8] P. C. Reeves-Hall and J. R. Taylor, "Wavelength and duration tunable sub-picosecond source using adiabatic Raman compression," Electron. Lett., vol. 37, pp. 417-418, 2001.

[9] K. Suzuki, K. Iwatsuki, S. Nishi, M. Saruwatari, K. Sato, and K. Wakita, " 2.5 ps soliton pulse generation at $15 \mathrm{GHz}$ with monolithically integrated MQW-DFB-LD/MQW-EA modulator and dispersion decreasing fiber," Electron. Lett., vol. 29, pp. 1713-1714, 1993.

[10] T. E. Murphy, "10-GHz 1.3-ps pulse generation using chirped soliton compression in a Raman gain medium," IEEE Photon. Technol. Lett., vol. 14, pp. 1424-1426, Oct. 2002

[11] M. D. Pelusi, Y. Matsui, and A. Suzuki, "Pedestal suppression from compressed femtosecond pulses using a nonlinear fiber loop mirror," IEEE J. Quantum Electron., vol. 35, pp. 867-874, June 1999.

[12] K. R. Tamura and M. Nakazawa, "A polarization-maintaining pedestal-free femtosecond pulse compressor incorporating an ultrafast dispersion-imbalanced nonlinear optical loop mirror," IEEE Photon. Technol. Lett., vol. 13, pp. 526-528, May 2001.

[13] M. Tadakuma, O. Aso, M. Sakano, and S. Namiki, " $100 \mathrm{GHz} 380$ fs pedestal suppressed pulse train generation by incorporating dispersionimbalanced nonlinear optical loop mirror into short comb-like dispersion profiled fiber," in Proc. 7th Optoelectronics Communications Conf. (OECC'2002), Yokohama, Japan, 2002, pp. 646-647.

[14] A. F. Evans, "Dispersion-tapered fiber in nonlinear loop mirrors for intensity and pulse width switching," in Proc. 22nd Eur. Conf. Optical Communication (ECOC'96), Oslo, Norway, 1996, Paper ThB.1.7.
[15] W. Cao and P. K. A. Wai, "Soliton-like pulse train generation using a nonlinear optical loop mirror constructed from dispersion decreasing fiber," IEEE Photon. Technol. Lett., vol. 14, pp. 1427-1429, Oct. 2002.

[16] D. J. Richardson, R. P. Chamberlin, L. Dong, D. N. Payne, A. D. Ellis, T. Widdowson, and D. M. Spirit, "Demonstration of $205 \mathrm{~km}$ transmission of $35 \mathrm{GHz}, 5$ ps pulses generated from a diode-driven, low jitter, beat signal to soliton train conversion source," Electron. Lett., vol. 31, pp. 470-472, 1995.

[17] M. D. Pelusi and H. F. Liu, "Higher order soliton compression in dispersion decreasing fibers," IEEE J. Quantum Electron., vol. 33, pp. 1430-1439, Aug. 1997.

[18] G. P. Agrawal, Nonlinear Fiber Optics, 2nd ed. New York: Academic, 1995, pp. 43-55.

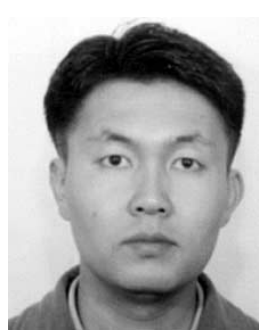

Ju Han Lee (S'03) received the B.S. and the M.S. degrees, both in electronics engineering, from Seoul National University, Seoul, Korea, in 1995 and 1998, respectively. He received the $\mathrm{Ph} . \mathrm{D}$. degree from Optoelectronics Research Centre (ORC), University of Southampton, Southampton, U.K., in May 2003.

From 1999 to 2000, he was with Korea Venture Creative Investment (KVCi) Inc., as an Analyst, where he was involved in analysis and investment on telecommunication technology. He joined the ORC in May 2000 and was a Visiting Researche with the COM center at Technical University of Denmark, Denmark, from July 2002 to September 2002. His research interests include optical fiber amplifiers (EDFA, Raman Amp.), nonlinear all-optical switching, optical code-division multiplexing systems, optical time-division multiplexing systems, holey fiber, and nonlinear fiber optics. He is an author or coauthor of more than 50 journal and conference technical papers.

Dr. Lee is a member of the Optical Society of America (OSA).

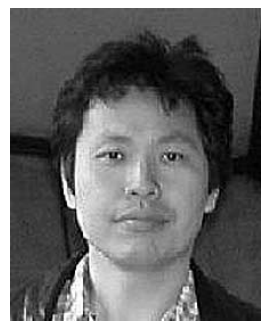

Taichi Kogure (M'99) was born in Chiba, Japan, on December 5, 1965. He received the B.S. degree in physics from Saitama University, Saitama, Japan, in 1991.

He joined Mitsubishi Electric Corporation, Kamakura, Japan, in 1991, where he has been engaged in research and development on laser diode modules, optical fiber amplifiers, and long-haul optical transmission systems. In 2003, he was on leave at the University of Southampton, Southampton, U.K.

Mr. Kogure is a member of the Institute of Electronics, Information and Communication Engineers (IEICE) of Japan.

David J. Richardson was born in Southampton, U.K., in 1964. He received the B.Sc. and Ph.D. degrees from Sussex University, Sussex, U.K., in 1985 and 1989 , respectively.

$\mathrm{He}$ is currently Deputy Director of the Optoelectronics Research Centre, Southampton University, Southampton, U.K. His research interests include holey fibers, high-power fiber lasers, short-pulse lasers, optical fiber communications, all-optical processing and switching, nonlinear optics, and the physics and applications of microstructured nonlinear/linear media. He has published more than 350 conference and journal papers in 15 years at ORC and produced over 15 patents. 